\title{
Nasal continuous positive airway pressure in stroke patients with sleep apnoea: a randomized treatment study
}

\author{
O. Sandberg*, K.A. Franklin*, G. Bucht*, S. Eriksson*, Y. Gustafson*
}

Nasal continuous positive airway pressure in stroke patients with sleep apnoea: a randomized treatment study. O. Sandberg, K.A. Franklin, G. Bucht, S. Eriksson, Y. Gustafson. (C) ERS Journals Ltd 2001.

ABSTRACT: The authors have investigated whether treatment of sleep apnoea with nasal continuous positive airway pressure (nCPAP) improves depressive symptoms, personal activities of daily living (ADL), cognitive functioning and delirium in patients that have suffered a stroke.

Sixty-three patients consecutively admitted to a stroke rehabilitation unit 2-4 weeks after a stroke, with an apnoea/hypopnoea index $\geqslant 15$, were randomized to either nCPAP treatment $(n=33)$ or a control group $(n=30)$. Four patients dropped out after randomization. Both groups were assessed at baseline and after 7 and 28 nights using the Montgomery-Åsberg Depression Rating Scale (MADRS), Barthel-ADL index, and the Mini-Mental State Examination (MMSE) scale.

Compared to the control group, depressive symptoms (MADRS total score) improved in patients randomized to nCPAP treatment $(\mathrm{p}=0.004)$. No significant treatment effect was found with regard to delirium, MMSE or Barthel-ADL index. Delirium and low cognitive level (MMSE score) explained poor compliance with nCPAP.

Depressive symptoms are reduced through nasal continuous positive airway pressure treatment in patients with severe stroke and sleep apnoea. Compliance with nasal continuous positive airway pressure treatment is a problem in stroke patients, especially when delirium and severe cognitive impairment occur.

Eur Respir J 2001; 18: 630-634.
*Dept of Community Medicine and Rehabilitation, Geriatric Medicine, and ${ }^{\#}$ Dept of Public Health and Clinical Medicine, Respiratory Medicine, Umeå University, Umeå, Sweden.

Correspondence: O. Sandberg, Sunderby Hospital, Dept of Geriatric Medicine and Rehabilitation, SE-971 80 Luleå, Sweden.

Fax: 46920283695

Keywords: Activities of daily living cerebrovascular disorders depression

nasal continuous positive airway pressure randomized clinical trials sleep apnoea syndromes

Received: August 32000 Accepted after revision May 102001

This study received support in the form of grants from the Gun and Bertil Stohne Foundation, Borgerskapets in Umeå Foundation, the 1987 Foundation for Stroke Research, the Fund for Medical Research at Umeå University Hospital, the Foundation for Gamla Tjänarinnor, the Thureús Fund and the Loo and Hans Osterman Fund.
Sleep apnoea is common among stroke patients; it is reported in as many as $44-74 \%$ of patients after a stroke [1-4]. Psychiatric symptoms, such as depression, depressive symptoms and anxiety, are also common among these patients $[5,6]$.

Nocturnal hypoxaemia, sleep fragmentation and impairment of cerebral perfusion during obstructive apnoeas have been suggested as the causes of daytime sleepiness and neuropsychological manifestations in patients with sleep apnoea [7-10]. To the authors' knowledge, no treatment studies of sleep apnoea in stroke patients have yet been published.

The present authors aimed to investigate whether treatment of sleep apnoea with nasal continuous positive airway pressure (nCPAP) improves depressive symptoms, personal activities of daily living (ADL), cognitive functioning, and delirium in patients that have suffered a stroke.

\section{Patients and methods}

Sixty-three out of 151 consecutive patients referred to a geriatric stroke rehabilitation unit 2-4 weeks after a stroke had an apnoea/hypopnoea index (AHI) $\geqslant 15$ according to overnight sleep apnoea recordings. These 63 patients were randomized through lots drawn by a person not involved in the study, with a $50 \%$ chance of being randomized to either nCPAP treatment for 4 weeks or to a control group without treatment. Four patients dropped out after randomization, two in the treatment and two in the control group. The clinical characteristics of the remaining 59 patients are presented in table 1 .

The study was approved by the Ethics Committee of the Medical Faculty of Umeå University, Umeå, Sweden (no. 95-013). Informed consent was obtained from all patients or their relatives before the study began, and patients in the control group were offered treatment with nCPAP after completion of the study.

\section{Overnight recordings}

The overnight recordings were made in hospital and sampled using a Micro Digitrapper SAS (Synectics 
Table 1. - Comparison at baseline of treatment and control groups, both with an apnoea/hypopnoea index $\geqslant 15$

\begin{tabular}{|c|c|c|c|}
\hline & $\begin{array}{l}\text { Treatment } \\
\text { group }\end{array}$ & $\begin{array}{l}\text { Control } \\
\text { group }\end{array}$ & p-value \\
\hline Patients $n$ & 31 & 28 & \\
\hline \multicolumn{4}{|l|}{ Age yrs } \\
\hline Mean & $78.1 \pm 6.4$ & $76.8 \pm 7.9$ & 0.504 \\
\hline Range & 61-89 & $52-86$ & \\
\hline \multicolumn{4}{|l|}{ Sex } \\
\hline Female & 17 & 15 & 0.922 \\
\hline Male & 14 & 13 & 0.922 \\
\hline BMI $\mathrm{kg} \cdot \mathrm{m}^{-2}$ & $24.5 \pm 4.1$ & $24.8 \pm 4.8$ & 0.798 \\
\hline \multicolumn{4}{|l|}{ Previous stroke } \\
\hline Cerebral infarction & 12 & 11 & 0.964 \\
\hline Intracerebral haemorrhage & 1 & 1 & 1.000 \\
\hline \multicolumn{4}{|l|}{ Current stroke } \\
\hline Cerebral infarction & 28 & 23 & 0.458 \\
\hline Thrombotic infarction & 15 & 15 & 0.691 \\
\hline Embolic infarction & 13 & 8 & 0.284 \\
\hline Intracerebral haemorrhage & 3 & 5 & 0.458 \\
\hline \multicolumn{4}{|l|}{ Neurological impairment } \\
\hline Hemiparesis left & 13 & 16 & 0.243 \\
\hline Hemiparesis right & 15 & 9 & 0.205 \\
\hline Aphasia & 3 & 5 & 0.458 \\
\hline Facial palsy left & 9 & 9 & 0.795 \\
\hline Facial palsy right & 7 & 5 & 0.653 \\
\hline \multicolumn{4}{|l|}{ Concurrent diseases } \\
\hline Ischaemic heart disease & 23 & 19 & 0.592 \\
\hline Cardiac arrhythmias & 21 & 13 & 0.098 \\
\hline Congestive heart failure & 18 & 16 & 0.943 \\
\hline Hypertension & 20 & 17 & 0.763 \\
\hline Diabetes mellitus & 10 & 12 & 0.401 \\
\hline COPD & 0 & 4 & 0.045 \\
\hline Previous diagnosed dementia & 1 & 1 & 1.000 \\
\hline Faecal incontinence & 9 & 10 & 0.583 \\
\hline Urinary incontinence & 16 & 15 & 0.880 \\
\hline Indwelling urinary catheter & 6 & 6 & 0.843 \\
\hline \multicolumn{4}{|l|}{ Outcome variables } \\
\hline Delirium & 22 & 24 & 0.172 \\
\hline MADRS & $21.0 \pm 10.4$ & $20.5 \pm 11.9$ & 0.860 \\
\hline MMSE & $16.6 \pm 7.4$ & $15.4 \pm 8.9$ & 0.582 \\
\hline Barthel-ADL index & $8.4 \pm 6.2$ & $7.5 \pm 6.0$ & 0.594 \\
\hline
\end{tabular}

Data are presented as number (n), range or mean \pm SD. BMI: body mass index; COPD: chronic obstructive pulmonary disease; MADRS: Montgomery-Åsberg Depression Rating Scale; MMSE: Mini-Mental State Examination; ADL: activities of daily living.

AB, Stockholm, Sweden) [11]. The recordings included nasal and oral airflow (3-port thermistor, Nihon Kohdeh Ze-732A, Tokyo, Japan), respiratory movements (Resp-EZ, EPM Systems, Midlothian, VA, USA), respiratory and body movements using a pressure-sensitive bed (PVDF motion sensor, Duorec Ltd, Turku, Finland), oxygen saturation and heart rate by finger oximetry (Nonin Medical Inc., Plymouth, MN, USA), body position, and a microphone placed on the throat.

All recordings were scored manually. The duration of sleep was estimated from the pressure-sensitive bed recordings [12] and from visual observation of the patients during the night. An obstructive apnoea was defined as cessation of airflow for $\geqslant 10 \mathrm{~s}$ with respiratory movements continuing during apnoea [13]. An obstructive hypopnoea was defined as a $50 \%$ decrease in the thermistor tracing in combination with an oxygen desaturation of $\geqslant 3 \%$ [14]. A central apnoea was defined as cessation of the thermistor tracing without any respiratory movements. A central hypopnoea was defined as a $50 \%$ decrease in the thermistor tracing with concomitant reduction of respiratory movements. AHI was defined as mean number of apnoeas and hypopnoeas per hour of sleep.

\section{Assessment scales}

Assessment scales were completed before the overnight recordings, and 7 and 28 nights, respectively, after starting nCPAP treatment. The control group completed the assessment scales before the initial overnight recording, and 7 and 28 nights later.

Depressive symptoms were assessed using the Montgomery-Åsberg Depression Rating Scale (MADRS). The maximum possible score was 60 points, which corresponds to severe depressive symptoms [15].

Personal ADL was assessed by two occupational therapists using the Barthel-ADL index, a 20-point scale in which 0 represents total personal ADL dependence [16].

Cognition was assessed using the Mini-Mental State Examination (MMSE) scale, scoring from 0-30 points, where 0 indicates severe cognitive decline. The test-retest reliability of the MMSE assessed by Pearson correlation is $0.83-0.99$, and concurrent validity by Pearson is $0.66-0.78$ [17].

Delirium was diagnosed according to the Diagnostic and Statistical Manual of Mental Disorders (DSM-IV) criteria [18].

\section{Nasal continuous positive airway pressure treatment}

Using REMstar Choice, (Respironics Inc., Pittsburgh, PA, USA), the mean nCPAP pressure was $5.8 \pm$ $1.4 \mathrm{cmH}_{2} \mathrm{O}$ (range 5.5-8.0). Patients were fitted with an appropriate mask and supplied with comfortable air pressure during the daytime. The pressure was adjusted until normal nocturnal finger-oximetry was obtained. The treatment was arbitrarily defined as adequate if the patient tolerated nCPAP for more than a mean of $4 \mathrm{~h}$ per night, during 28 nights.

\section{Statistical analysis}

Univariate analysis of differences between treatment and control group were performed with an unpaired t-test and Pearson's Chi-squared test. Data are presented as mean \pm SD. Fisher's exact test was used when the expected cell numbers were less than five.

Data from 31 of 708 assessments of delirium, MADRS, MMSE and Barthel-ADL index in the 59 patients were missing. Five patients in the treatment group and three in the control group had some missing data. The missing data were replaced with mean values calculated for each outcome variable, i.e. delirium, MADRS total score, MMSE score, and 
Table 2. - Comparison of baseline sleep results between study and control groups

\begin{tabular}{lccc}
\hline & $\begin{array}{c}\text { Treatment } \\
\text { group }\end{array}$ & $\begin{array}{c}\text { Control } \\
\text { group }\end{array}$ & p-value \\
\hline Patients n & 31 & 28 & \\
Total sleep time h & $8.2 \pm 1.3$ & $7.8 \pm 1.5$ & 0.304 \\
AHI & $32 \pm 11$ & $29 \pm 13$ & 0.387 \\
AHI supine & $36 \pm 13$ & $30 \pm 16$ & 0.102 \\
AHI nonsupine & $11 \pm 14$ & $13 \pm 20$ & 0.650 \\
Mean apnoea time s & $27 \pm 7$ & $25 \pm 8$ & 0.338 \\
Nadir mean $\mathrm{Sa}_{\mathrm{a}, \mathrm{O}_{2}} \%$ & $78 \pm 10$ & $79 \pm 8$ & 0.827 \\
\hline
\end{tabular}

Data are presented as mean \pm SD unless otherwise stated. AHI: apnoea/hypopnoea index; $S_{\mathrm{a}_{2} \mathrm{O}_{2}}$ : arterial oxygen saturation.

Barthel-ADL index score, in the treatment and control group at baseline, and after 7 and 28 nights, respectively.

The dependent variables in the treatment group were assessed as the differences between pretreatment values and the mean of values after 7 and 28 nights, respectively, compared with the corresponding difference in the control group.

A multivariate analysis of the variance with repeated measurements, including contrast analysis, was used for statistical analysis.

A multiple linear regression model was used to investigate whether the improvement of depressive symptoms could be explained by the use of antidepressants. Delta values for MADRS score were used as dependent variables and the authors controlled for sleep apnoea.

A p-value of $<0.05$ was considered statistically significant.

\section{Results}

The treatment and control groups did not differ significantly at baseline with regard to delirium, MADRS total score, MMSE score, Barthel-ADL index, overnight respiratory sleep recording results (table 2) or in the other variables presented in table 1, except for chronic obstructive pulmonary disease, which was present in four control group patients. The type of stroke, stroke location and type of apnoeas (i.e. obstructive or central) did not differ between treatment and control group.

The median of the total AHI (both central and obstructive) for the studied sample $(n=59)$ was 28 (range 15-79) (interquartile range 20-39). The median central AHI was 15 (range 0-74) (interquartile range 1-31), and the median obstructive AHI was 7 (range 0-43) (interquartile range 1-21).

After 28 nights of nCPAP treatment, depressive symptoms (MADRS total score) in the treatment group improved significantly compared to the control group ( $\mathrm{p}=0.004)$, according to a multivariate analysis of the variance with repeated measurements including contrast analysis (table 3). The MADRS total score decreased by a mean of 5.4 points $(25.7 \%)$ in treated subjects $(n=31)$, between baseline and follow-up after 28 nights. The corresponding figure for controls $(n=28)$ was a mean increase of 1.8 points $(8.1 \%)$ $(\mathrm{p}=0.032)$ in MADRS total score. No significant treatment effect was found with regard to delirium, MMSE or Barthel-ADL index.

Eight of 59 stroke patients were receiving antidepressant treatment at baseline (three in the treatment group and five in the control group). Antidepressant treatment was initiated in another three patients during the course of the study (two in the treatment group and one in the control group). Multiple linear regression showed that treatment with antidepressants did not influence the MADRS total score in the treatment and control groups.

The patients in the treatment group used the nCPAP for a mean of $4.1 \pm 3.6 \mathrm{~h}$ (range $0-10.9 \mathrm{~h}$ ). Sixteen of 31 patients in the treatment group used nCPAP for more than an average of $4 \mathrm{~h}$ per night. The remaining 15 patients had a low compliance to nCPAP in that they used it for less than a mean of $4 \mathrm{~h}$ per night. Patients who complied with treatment for $>4 \mathrm{~h}$ per night had less delirium $(\mathrm{p}<0.001)$, were less depressed (MADRS) $(\mathrm{p}=0.041)$, had a higher

Table 3. - Scores for delirium, depressive symptoms, cognitive function and activities of daily living (ADL) in treatment and control groups

\begin{tabular}{lcccc}
\hline & Baseline day 1 & $\begin{array}{c}\text { Difference } \\
\text { night 7-day 1 }\end{array}$ & $\begin{array}{c}\text { Difference } \\
\text { night 28-day 1 }\end{array}$ & $\begin{array}{c}\text { p-value by contrast } \\
\text { analysis }\end{array}$ \\
\hline $\begin{array}{l}\text { Delirium \% } \\
\text { Treatment }\end{array}$ & 71.0 & & & 0.881 \\
$\begin{array}{c}\text { Control } \\
\text { MADRS }\end{array}$ & 85.7 & -17.2 & -15.4 & 0.004 \\
$\quad \begin{array}{l}\text { Treatment } \\
\text { Control }\end{array}$ & $21.0(17.6-24.7)$ & $-5.0(-8.2--1.8)$ & $-5.4(-9.7--1.1)$ & 0.744 \\
$\begin{array}{l}\text { MMSE } \\
\text { Treatment }\end{array}$ & $20.5(16.0-25.0)$ & $2.6(0.1-5.1)$ & $1.8(-1.5-5.1)$ & \\
$\quad$ Control & $16.6(13.9-19.3)$ & $1.4(0.2-2.6)$ & $2.6(1.1-4.1)$ & 0.980 \\
Barthel-ADL & $15.4(12.0-18.8)$ & $0.9(0.0-1.8)$ & $2.8(1.2-4.4)$ & \\
$\quad$ Treatment & $8.4(6.2-10.6)$ & $1.5(0.5-2.5)$ & $1.1(-0.4-2.6)$ & \\
$\quad$ Control & $7.5(5.2-9.8)$ & $1.0(0.2-1.8)$ & $1.1(-0.3-2.5)$ & \\
\hline
\end{tabular}

Data are presented as mean (95\% confidence interval) unless otherwise stated. MADRS: Montgomery-Åsberg Depression Rating Scale; MMSE: Mini-Mental State Examination. "\#: treatment group compared to control group. 
cognitive level (MMSE) ( $p=0.018$ ), and had less urinary incontinence $(\mathrm{p}<0.05)$ at baseline, compared to those who complied with treatment for $<4 \mathrm{~h}$ per night. Patients with good and poor compliance did not differ with regard to type of stroke, stroke location, hemiparesis, aphasia, facial palsy, type of apnoeas (i.e. obstructive or central), mean AHI, MADRS or Barthel-ADL index at baseline.

\section{Discussion}

Depressive symptoms improved significantly in stroke patients with an $\mathrm{AHI} \geqslant 15$, randomized to nCPAP treatment during 28 nights.

Depression affects $30-60 \%$ of patients in the acute phase after stroke [5]. It impairs the rehabilitation process and, therefore, the functional recovery of patients $[19,20]$. The MADRS is particularly sensitive to treatment effects [15] and it has been shown to be the best examiner rating scale for assessing depressive symptoms in elderly stroke patients [21]. Whether the improvement in depressive symptoms in the present patients is an effect of improved nocturnal oxygenation, a decreased number of nocturnal arousals, or normalization of cerebral haemodynamics, cannot be determined from this study.

The Barthel-ADL index [16] is limited in scope and may not detect low levels of disability; the scale steps are not equal and the scale is insensitive to change [22], which might be a reason why no significant effect of nCPAP treatment on ADL was seen.

Nasal CPAP treatment did not improve cognitive functioning according to the MMSE scale, but this scale is not very sensitive to change [17]. Neuropsychological tests are better, although it was not possible to apply them due to the patients' multiple handicaps.

Two case studies have reported reversibility of delirium after treatment with nCPAP [23, 24]. The present study did not confirm these preliminary findings, nor did the authors find any significant effects of nCPAP treatment on MMSE, or BarthelADL index. However, calculation shows that the power of this study to detect changes in delirium or MMSE is $<17 \%$, and to detect treatment effects on these variables would require a much larger study.

Delirium and low cognitive level (MMSE score) explained the low compliance with nCPAP treatment. Thus, the ability of a patient to accept nCPAP treatment for more than a mean of $4 \mathrm{~h}$ per night might reflect a less severe stroke.

The simplified sleep recordings and nCPAP titration, using only oximetry rather than polysomnography, are below the current standard of many sleep laboratories around the world. The nCPAP can be criticized since apnoeas without desaturation may persist with the titration method used. However, the results are clear and it is possible that they would have been even better if an up-to-date method for optimized nCPAP titration had been used.

In conclusion, depressive symptoms are reduced with nasal continuous positive airway pressure treatment in patients with severe stroke and sleep apnoea. Compliance with nasal continuous positive airway pressure treatment is, however, a problem in stroke patients, especially when accompanied by delirium and severe cognitive impairment.

\footnotetext{
Acknowledgements. The authors thank U.B. Johansson for data collection and C. SahlinIngridsson for scoring the sleep apnoea recordings. Further thanks go to L. Stoltz and M. Larsson for activities of daily living assessments.
}

\section{References}

1. Bassetti C, Aldrich M. Sleep apnea in acute cerebrovascular diseases: Final report on 128 patients. Sleep 1999; 22: 217-223.

2. Wessendorf TE, Teschler H, Wang YM, Konietzko N, Thilmann AF. Sleep-disordered breathing among patients with first-ever stroke. J Neurol 2000; 247: 41-47.

3. Parra O, Arboix A, Bechich S, et al. Time course of sleep-related breathing disorders in first-ever stroke or transient ischemic attack. Am J Respir Crit Care Med 2000; 161: 375-380.

4. Sandberg O, Franklin KA, Bucht G, Gustafson Y. Sleep apnea, delirium, depressed mood, cognition, and ADL ability after stroke. J Am Geriatr Soc 2001; 49: 391-397.

5. Gustafson Y, Nilsson I, Mattsson M, Åström M, Bucht G. Epidemiology and treatment of post-stroke depression. Drugs Aging 1995; 7: 298-309.

6. Derderian SS, Bridenbaugh RH, Rajagopal KR. Neuropsychologic symptoms in obstructive sleep apnea improve after treatment with nasal continuous positive airway pressure. Chest 1988; 94: 10231027.

7. Bédard M-A, Montplaisir J, Richer F, Rouleau I, Malo J. Obstructive sleep apnea syndrome: pathogenesis of neuropsychological deficits. J Clin Exp Neuropsychol 1991; 13: 950-964.

8. Engleman HM, Martin SE, Deary IJ, Douglas NJ. Effect of CPAP therapy on daytime function in patients with mild sleep apnoea/hypopnoea syndrome. Thorax 1997; 52: 114-119.

9. Bålfors EM, Franklin KA. Impairment of cerebral perfusion during obstructive sleep apneas. Am J Respir Crit Care Med 1994; 150: 1587-1591.

10. Franklin KA, Sandström E, Johansson G, Bålfors EM. Hemodynamics, cerebral circulation and oxygen saturation in cheyne-stokes respiration. J Appl physiol 1997; 83: 1184-1191.

11. Zucconi M, Ferini-Strambi L, Castronovo V, Oldani A, Smirne S. An unattended device for sleep-related breathing disorders: validation study in suspected obstructive sleep apnoea syndrome. Eur Respir $J$ 1996; 9: 1251-1256.

12. Svanborg E, Larsson H, Carlsson-Nordlander B, Pirskanen R. A limited diagnostic investigation for obstructive sleep apnea syndrome. Oximetry and static charge sensitive bed. Chest 1990; 98: 1341-1345.

13. Keenan Bornstein S. Respiratory monitoring during sleep: polysomnography. In: Guilleminault C, ed. Sleeping and Waking Disorders: Indications and Techniques. Menlo Park, Addison Wesley, 1982; pp. 183-212. 
14. American Sleep Disorders Association. Intrinsic sleep disorders. In: Thorpy MJ, ed. The International Classification of Sleep Disorders. Diagnostic and Coding Manual. Lawrence, Kansas, Allan Press, 1990; pp. 52-61.

15. Montgomery SA, Åsberg M. A new depression scale designed to be sensitive to change. Br J Psychiatry 1979; 134: 382-389.

16. Mahoney FI, Barthel DW. Functional evaluation: The Barthel index. Md Med $J$ 1965; 14: 61-65.

17. Folstein MF, Folstein SE, McHugh PR. Mini-Mental State: a practical method for grading the cognitive state of patients for the clinician. J Psychiatr Res 1975; 12: $189-198$.

18. American Psychiatric Association. Diagnostic and Statistical Manual of Mental Disorders. 4th Edn (DSM-IV). Washington, D.C., American Psychiatric Association, 1994.

19. Morris LP, Raphael B, Robinson RG. Clinical depression is associated with impaired recovery from stroke. Med J Aust 1992; 157: 239-242.

20. Parikh RM, Robinson RG, Lipsey JR, Starkstein SE, Fedoroff JP, Price TR. The impact of poststroke depression on recovery in activities of daily living over a 2-year follow-up. Arch Neurol 1990; 47: 785-789.

21. Agrell B, Dehlin O. Comparison of six depression rating scales in geriatric stroke patients. Stroke 1989; 20: 1190-1194.

22. McDowell I, Newell C. Measuring Health. A Guide to Rating Scales and Questionnaires. New York, Oxford University Press, 1996.

23. Whitney JF, Gannon DE. Obstructive sleep apnea presenting as acute delirium. Am J Emerg Med 1996; 14: 270-271.

24. Munoz X, Marti S, Sumalla J, Bosch J, Sampol G. Acute delirium as a manifestation of obstructive sleep apnea syndrome. Am J Respir Crit Care Med 1998; 158: $1306-1307$. 